\title{
An Image Quality Comparison Study Between Homemade and Commercial Dental Cone-Beam CT Systems
}

\author{
Trang Thi Ngoc Tran ${ }^{1} \cdot$ David Shih-Chun $\mathrm{Jin}^{2} \cdot$ Kun-Long Shih ${ }^{3} \cdot$ Ming-Lun Hsu ${ }^{1} \cdot$ Jyh-Cheng Chen ${ }^{2,4,5}$
}

Received: 26 January 2021 / Accepted: 28 September 2021 / Published online: 15 November 2021

(c) The Author(s) 2021

\begin{abstract}
Purpose Cone-beam computed tomography (CBCT) has been widely applied in dental and maxillofacial imaging. Several dental CBCT systems have been recently developed in order to improve the performance. This study aimed to evaluate the image quality of our prototype (YMU-DENT-P001) and compare with a commercial POYE Expert 3DS dental CBCT system (system A).

Methods The Micro-CT Contrast Scale, Micro-CT Water and Micro-CT HA phantoms were used to evaluate the two CBCT systems in terms of contrast-to-noise ratio (CNR), signal-to-noise ratio (SNR), uniformity (U), distortion, and linearity in the relationship between image intensity and calcium hydroxyapatite concentration. We also fabricated a proprietary thin-wire phantom to evaluate full width at half maximum (FWHM) spatial resolution. Both CBCT systems used the same exposure protocol, and data analysis was performed in accordance with ISO standards using a proprietary image analysis platform.

Results The SNR of our prototype system was nearly five times higher than that of system A (prototype: $159.85 \pm 3.88$; A: $35.42 \pm 0.61 ; p<0.05$ ) and the CNR was three times higher (prototype: $329.39 \pm 5.55 ; A: 100.29 \pm 2.31 ; p<0.05$ ). The spatial resolution of the prototype $(0.2446 \mathrm{~mm})$ greatly exceeded that of system A $(0.5179 \mathrm{~mm})$ and image distortion was lower (prototype: $0.03 \mathrm{~mm}$; system A: $0.285 \mathrm{~mm}$ ). Little difference was observed between the two systems in terms of the linear relationship between bone mineral density (BMD) and image intensity.

Conclusions Within the scope of this study, our prototype YMU-DENT-P001 outperformed system A in terms of spatial resolution, SNR, CNR, and image distortion.
\end{abstract}

Keywords Cone-beam computed tomography $(\mathrm{CBCT}) \cdot$ System performance $\cdot$ Image quality $\cdot$ Spatial resolution $\cdot$ Signal to noise ratio (SNR) · Contrast to noise ratio (CNR)

Ming-Lun Hsu

mlhsu@nycu.edu.tw

$\triangle$ Jyh-Cheng Chen

jyhcheng@nycu.edu.tw

1 Department of Dentistry, National Yang Ming Chiao Tung University, Taipei, Taiwan

2 Department of Biomedical Imaging and Radiological Sciences, National Yang Ming Chiao Tung University, Taipei, Taiwan

3 Department of Electro-Optical Engineering, National Taipei University of Technology, Taipei, Taiwan

4 Department of Medical Imaging and Radiological Technology, Yuanpei University of Medical Technology, Hsinchu, Taiwan

5 School of Medical Imaging, Xuzhou Medical University, Xuzhou, Jiangsu, China

\section{Introduction}

\subsection{Cone-Beam Computed Tomography}

Since it was first developed in the late 1990s, cone-beam computed tomography (CBCT) has been widely used to obtain multi-planar views of targeted anatomical or abnormal structures in dental and maxillofacial imaging [1,2]. A number of researchers have concluded that CBCT is comparable to multi-slice computed tomography (MSCT) in terms of image quality and the visibility of structures, while providing superior bone segmentation performance [3-5]. In clinical practice, the quality of these image can be assessed in terms of visibility, such as the ability to differentiate between tissue types, the ability to detect root fractures or bone fractures, and the ability to identify instances of pathology, cortical bone thickness, and the structure of trabecular 
bone. Dental image quality can also be measured quantitatively in terms of spatial resolution, contrast sensitivity, and noise $[6,7]$. Note that these factors are inter-dependent and should always be considered together in assessing image quality.

\subsection{Quantitative Assessment of Image Quality}

Image quality can be defined by many factors as imaging hardware, exposure settings, image acquisition and image reconstruction [6]. Spatial resolution indicates the ability to distinguish between small discrete objects or structures, which appreciates the fine details in an image. Some authors have described spatial resolution as the degree of sharpness determined by the two-dimensional detector (size, number and spacing of detector elements), the three-dimensional reconstruction process, the size of the X-ray focal spot, the source-object-detector distances, reconstruction filter, and reconstructed voxel size [6-8]. Spatial resolution is particularly important when dealing with highly complex anatomic structures and fine imperfections in the dental maxillofacial region (e.g., periodontal ligament gap, root fractures, and bone fractures).

Contrast in radiographic imaging indicates the ability to differentiate various material types which having different attenuation coefficients. Image contrast is determined by many factors as the contrast of physical objects or materials, exposure factor, the bit depth of the reconstructed image, and the display settings (e.g., window level) in the image visualization stage [7]. Compared to conventional medical CT systems, which have a very high contrast sensitivity, CBCT systems perform poorly in measuring mineral density and differentiating between soft tissue types [9]. The grey scale in CBCT is also represented using intensity values, unlike the CT contrast scale used in conventional CT system [10]. Moreover, manufacturers differ in their reported image pixel values, which makes it difficult to obtain quantitative comparisons in term of contrast sensitivity.

Image noise in CT images refers to random variability in voxel values, which manifests as graininess compromising the visibility of objects comprising low-contrast tissue. Noise levels can be reduced by altering the exposure settings, including the scanning time, tube amperage, and kilovoltage peak. During the reconstruction stage, it is also possible to use smoothing filters to reduce noise. However, in most practical situations, image quality is determined primarily by the lesion-to-background contrast, which relates more strongly to contrast-to-noise ratio (CNR) and signalto-noise ratio (SNR) than to image noise [11].

In this study, we compared the quality of images acquired using two CBCT systems: POYE Expert 3DS dental CBCT (Taipei, Taiwan) (system A) and a prototype system developed by the Department of Biomedical Imaging and
Radiological Sciences - National Yang Ming Chiao Tung University, Taiwan (YMU-DENT- P001).

\section{Materials and Methods}

\subsection{Phantoms and Image Acquisitions}

Four phantoms were utilized to obtain quantitative assessments of image quality: QRM Micro-CT water phantom, QRM Micro-contrast Scale phantom, Micro-CT HA phantom (QRM Quality Assurance in Radiology and Medicine $\mathrm{GmbH}$, Möhrendorf, Germany) and a thin-wire phantom fabricated in the current study (Table 1).

In both CBCT systems, the phantoms were scanned under the same exposure conditions: tube voltage $(60 \mathrm{kVp})$ and tube current $(2 \mathrm{mAs})$ with the phantoms placed at the isocenter. Technical differences between system A and Prototype YMU-DENT-P001 are listed in Table 2.

\subsection{Method of Analysis}

Acquired data was processed using an analysis platform designed by our lab in accordance with ISO15708-2:2002, pertaining to the interpretation of CT non-destructive images [13]. The CT image analysis protocol included five steps:

- Setting the format for input data and defining the volume of interest (VOI);

- Adjusting the window level to ensure that images displayed by the two systems are comparable;

- Image viewer adjusted to central or nearly central slices;

- Analysis function setup, including a list of analytic options;

- Exporting results.

The parameters used to describe image quality included signal-to-noise ratio (SNR), contrast-to-noise ratio (CNR), uniformity (U), distortion (roundness, diameter), linearity, and spatial resolution.

\subsubsection{SNR, U, and Distortion (Roundness and Diameter)}

The water phantom was used to calculate SNR, U, and distortion. The central or nearly central slices of the phantom was used in analyzing the region of interest (ROI). Five featureless circular ROIs were chosen in which the diameter of each ROI accounted for $20 \%$ of the diameter of the phantom (Fig. 1).

SNR was determined as the ratio of the average and standard deviation of all pixel intensity values in each ROI in accordance with ISO15708-2:2002 [13]. 


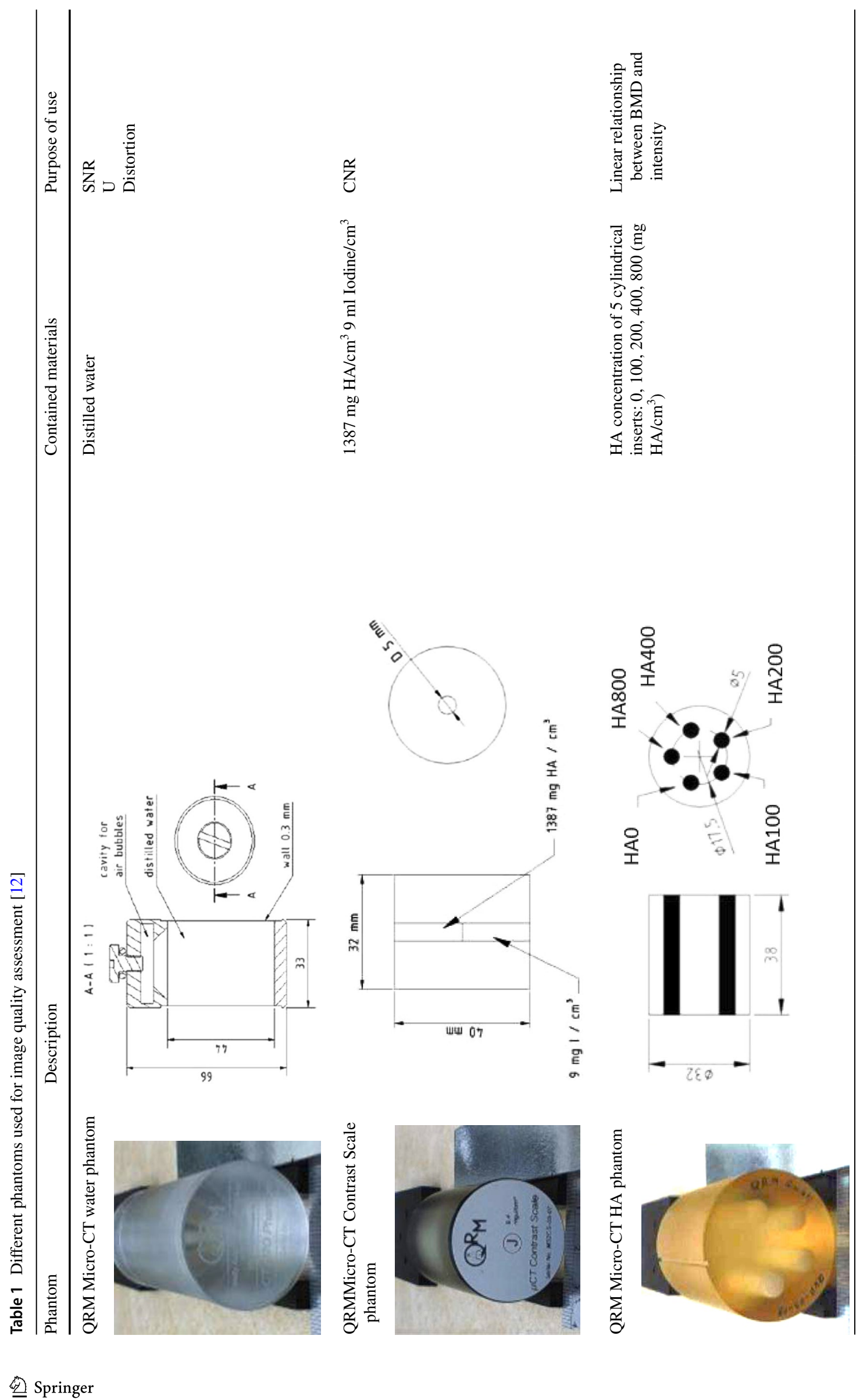




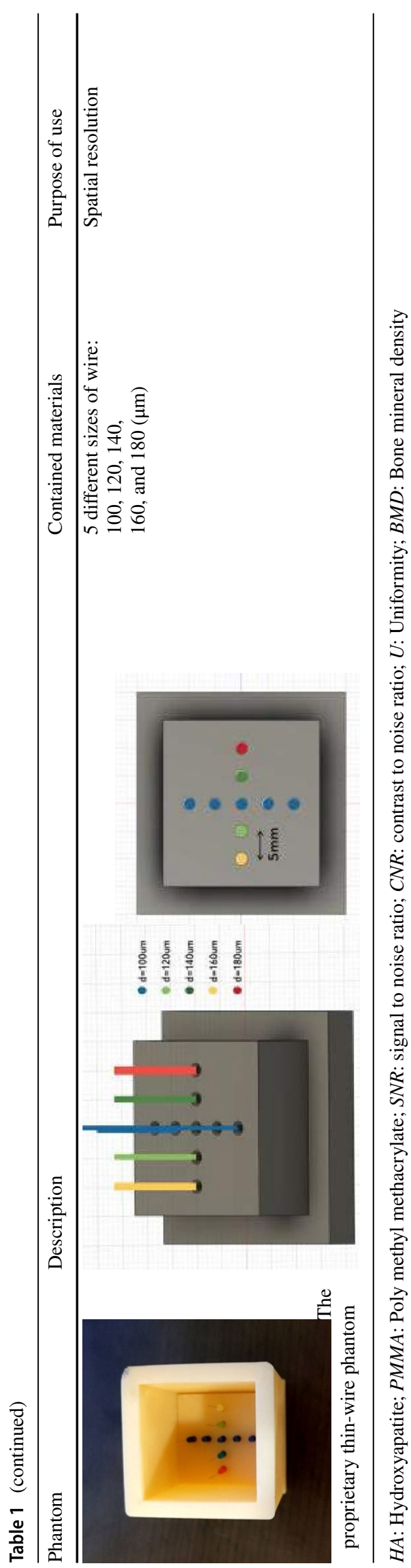

$S N R_{R O I(i)}=\frac{\mu_{R O I(i)}}{\sigma_{R O I(i)}}, i f$
$S N R=\frac{\sum_{1}^{5} S N R_{R O I(i)}}{5}$

The uniformity (U) of each ROI was expressed as follows:

$U_{R O I(i)}=\left(\frac{\mu_{\max }-\mu_{\min }}{\mu_{\max }+\mu_{\min }} * 100 \%\right)_{R O I(i)}$, ifrom 1 to 5

$U=\frac{\sum_{1}^{5} U_{R O I(i)}}{5}$

where $\mu_{\max }$ and $\mu_{\min }$ respectively indicate the maximum and minimum pixel intensity values. The average $U$ value of the five ROIs was then calculated. The lower of $U$ value, the better uniformity of the image.

Diameter was determined as the circumference of the ROI around its outline (Fig. 1).

As shown in Fig. 2, image deformity was defined in terms of roundness, which was measured in accordance with the out-of-roundness standard ANSI-B89.3.1 [14], as follows:

Roundness $=|d \max -d \min |$

\subsubsection{Linearity in the Relationship Between Image Intensity and Density}

The QRM Micro-CT HA phantom includes five cylindrical inserts differing in HA concentration $(0,100,200,400$ and $800 \mathrm{mg} \mathrm{HA} / \mathrm{cm}^{3}$ ). For each HA concentration, the ROI is a homogenous area within the test cylinder, accounting for $90 \%$ of the circular reconstructed area. The ROI diameter of the prototype was 49 pixels $(4.704 \mathrm{~mm})$ and the diameter of system A was 38 pixels $(4.75 \mathrm{~mm})$ (Fig. 3). The range and mean pixel value of each ROI were recorded.

A comparison of the two systems was performed by prescanning the HA phantom using a GE Discovery 670 CT scanner (GE Healthcare, Milwaukee, USA) to obtain reference HU values. Note that we used axial view with open view to obtain $60-120 \mathrm{kVp}$ CT data. Two linear regression functions were performed in accordance with the known BMD and clinical $\mathrm{HU}$ value to characterize the relationship between BMD and $\mathrm{HU}$ as well as the intensity.

$$
\left\{\begin{array}{c}
H U=y+1024=a \times \text { intensity }+b \\
\text { intensity }=c \times B M D+d
\end{array}\right.
$$

To the same BMD phantom, we applied the linear transfer and inverse transfer to normalize the values for comparison. 
Table 2 Comparison of technical parameters and reconstruction algorithms between two dental CBCT systems

\begin{tabular}{|c|c|c|}
\hline & System A & Prototype YMU-DENT-P001 \\
\hline SOD & $350 \mathrm{~mm}$ & $430 \mathrm{~mm}$ \\
\hline OID & $170 \mathrm{~mm}$ & $190 \mathrm{~mm}$ \\
\hline Rotation angle & $\begin{array}{l}\text { Start angle: } 135 \\
\text { Rotation angle: } 268\end{array}$ & $\begin{array}{l}360 \text { full rotation with angle sampling: } \\
2 \text { degree: QRM phantom } \\
\text { degree: customized phantom }\end{array}$ \\
\hline Sensor size & $\begin{array}{l}\text { Horizontal: } 960 \text { pixel } \\
\text { Vertical: } 786 \text { pixel }\end{array}$ & $\begin{array}{l}\text { Horizontal: } 2176 \text { pixel } \\
\text { Vertical: } 1192 \text { pixel }\end{array}$ \\
\hline Sensor element size & $0.15 \mathrm{~mm}$ & $0.139 \mathrm{~mm}$ \\
\hline Binned input & 1 & 1 \\
\hline Voxel size & $0.125 \mathrm{~mm}$ & $0.096 \mathrm{~mm}$ \\
\hline FOV & $\begin{array}{l}\text { Diameter: } 98 \mathrm{~mm} \\
\text { Height: } 80 \mathrm{~mm}\end{array}$ & $21.97 \mathrm{~cm}$ (horizontal)* $138 \mathrm{~cm}$ (vertical) \\
\hline X-ray source & $60-90 \mathrm{kV}, 2-10 \mathrm{~mA}$ & $50-90 \mathrm{kV}, 2-10 \mathrm{~mA}$ \\
\hline Focal spot & $0.5 \mathrm{~mm}$ (Toshiba, Japan) & $0.5 \mathrm{~mm}$ (PDM, Spellman, US) \\
\hline Detector & CMOS & CMOS \\
\hline Bit depth & $>14$ bit & 16 bit \\
\hline Source filtration & $2.6 \mathrm{~mm}$ Alumina & $3 \mathrm{~mm}$ Alumina \\
\hline Reconstruction algorithm & Analytical: FDK algorithm & Analytical: FDK algorithm \\
\hline Pre- reconstruction calibration & $\begin{array}{l}\text { Flood field correction; dark-field correction; gain cor- } \\
\text { rection; X-ray exposure uniformity; center of } \\
\text { rotation alignment }\end{array}$ & Flood field, dark field correction, rigid body alignment \\
\hline Post- reconstruction correction & $\begin{array}{l}\text { Energy-related attenuation coefficient based HU cor- } \\
\text { rection; ring-artifact reduction, outlier-voxel removal } \\
\text { (noise, spark points are included) and circle mask- } \\
\text { out of FOV region to be zero, and metal artifact } \\
\text { reduction }\end{array}$ & $\begin{array}{l}\text { Dynamic HU calibration, noise reduction, ring reduc- } \\
\text { tion and metal artifact reduction algorithm }\end{array}$ \\
\hline Software kernel & $\begin{array}{l}\text { AVRG-a commercialized algorithm supplied by } \\
\text { Orimtech (Illinois, USA) }\end{array}$ & $\begin{array}{l}\text { Self-developed software integrated by open-source } \\
\text { package to proof of concept on python platform } \\
\text { (TomoPy with ASTRA package) }\end{array}$ \\
\hline
\end{tabular}

$S O D$ : Source to object distance; $O I D$ : Object to imaging detector distance; FOV: Field of view; $C M O S$ : Complementary metal oxide semiconductor; FBP: Filtered back projection; FDK: Feldkamp-Davis-Kress; $H U$ : Hounsfield unit

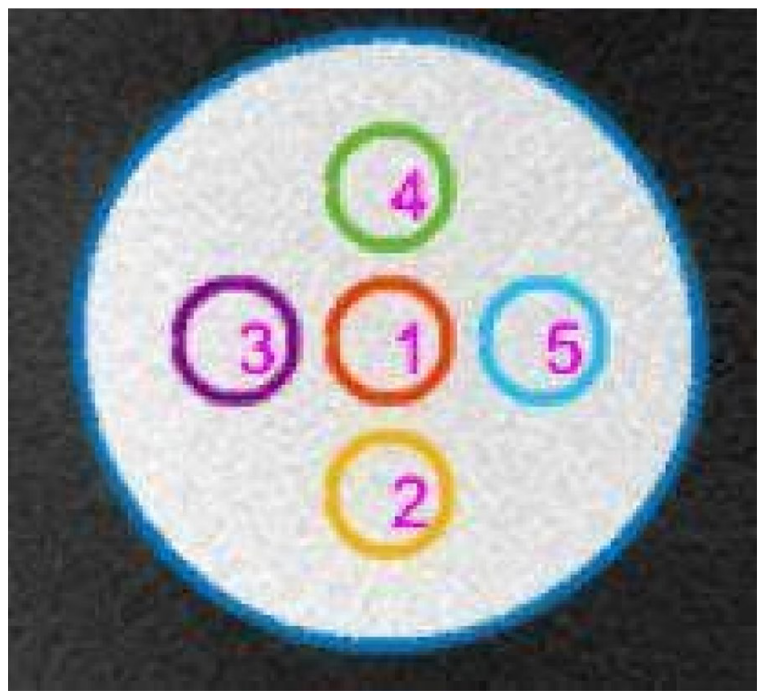

The final comparison was conducted using plots of intensity versus HU.

\subsubsection{Contrast-To-Noise Ratio (CNR)}

Central slices of the reconstructed QRM contrast-scale phantom were chosen. The ROI diameter was 52 pixels $(4.992 \mathrm{~mm})$ in the prototype system and 40 pixels $(5 \mathrm{~mm})$ in system A. We calculated average intensity values in the center (blue circle) as well as the background (red circles). CNR values are expressed in accordance with ISO157082:2002 standards (Fig. 4):

$C N R=\frac{\mu_{R O I 1}-\operatorname{mean}\left(\mu_{R O I(i)}\right)}{\sqrt{\sigma_{R O I 1}^{2}+\operatorname{mean}\left(\sigma_{R O I(i)}^{2}\right)}}, i$ from 2 to 5

Fig. 1 Regions of interest for signal-to-noise ratio (SNR) \& uniformity (U) analysis: from 1 to 5; region for distortion determination: outline blue circle 


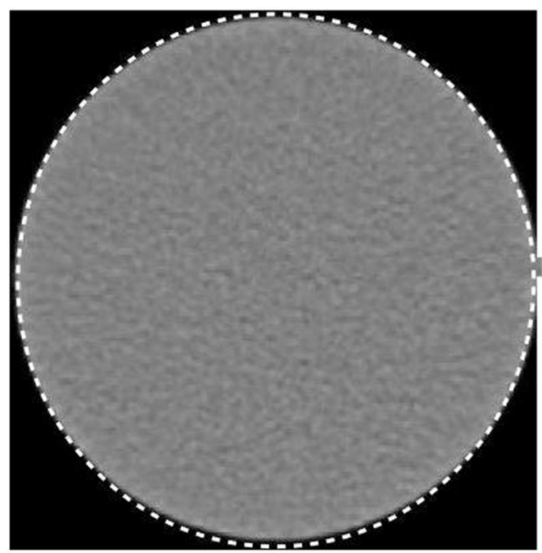

Fig. 2 Roundness calculation

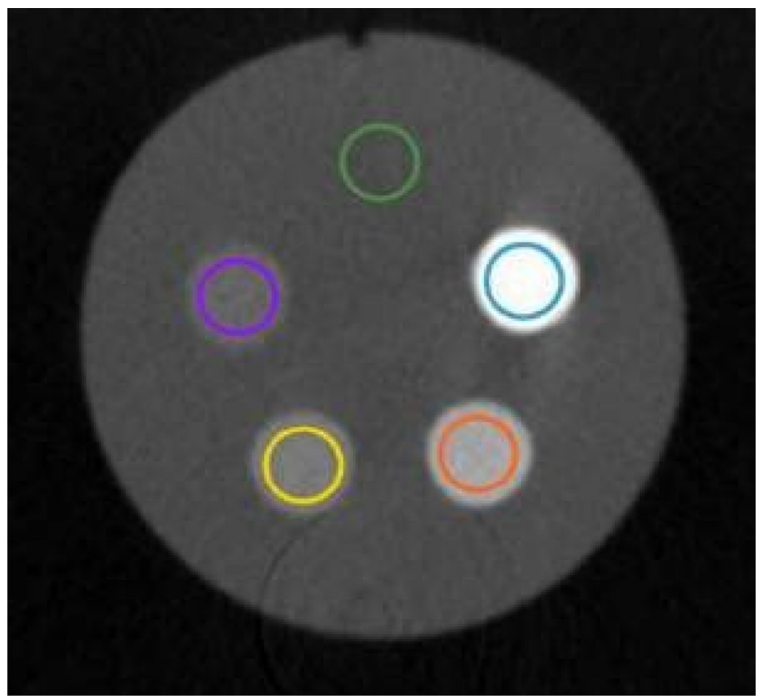

Fig. 3 Regions of interest determination for contrast-to-noise ratio

\subsubsection{Spatial Resolution}

As shown in Table 1, we created a proprietary wire phantom which comprised wires of various thicknesses running parallel to the axis of rotation and held in place using epoxy resin. The wires were laid out in a cross-shaped pattern. Five wires of various thicknesses were arranged from left to right along horizontal axis as follows: 160, 120, 100, 140, $180 \mu \mathrm{m}$. The resolution is better in central than in peripheral region, so we put the smallest wire $(100 \mu \mathrm{m})$ in the center and keep the same-size wires along the vertical axis. Our objective was to perform the point spread function (PSF) based on the distribution of CT pixel values vertically and horizontally along the midline of the reconstructed image. Gaussian fitting was then used to calculate the full width at half maximum (FWHM). The mean spatial resolution was
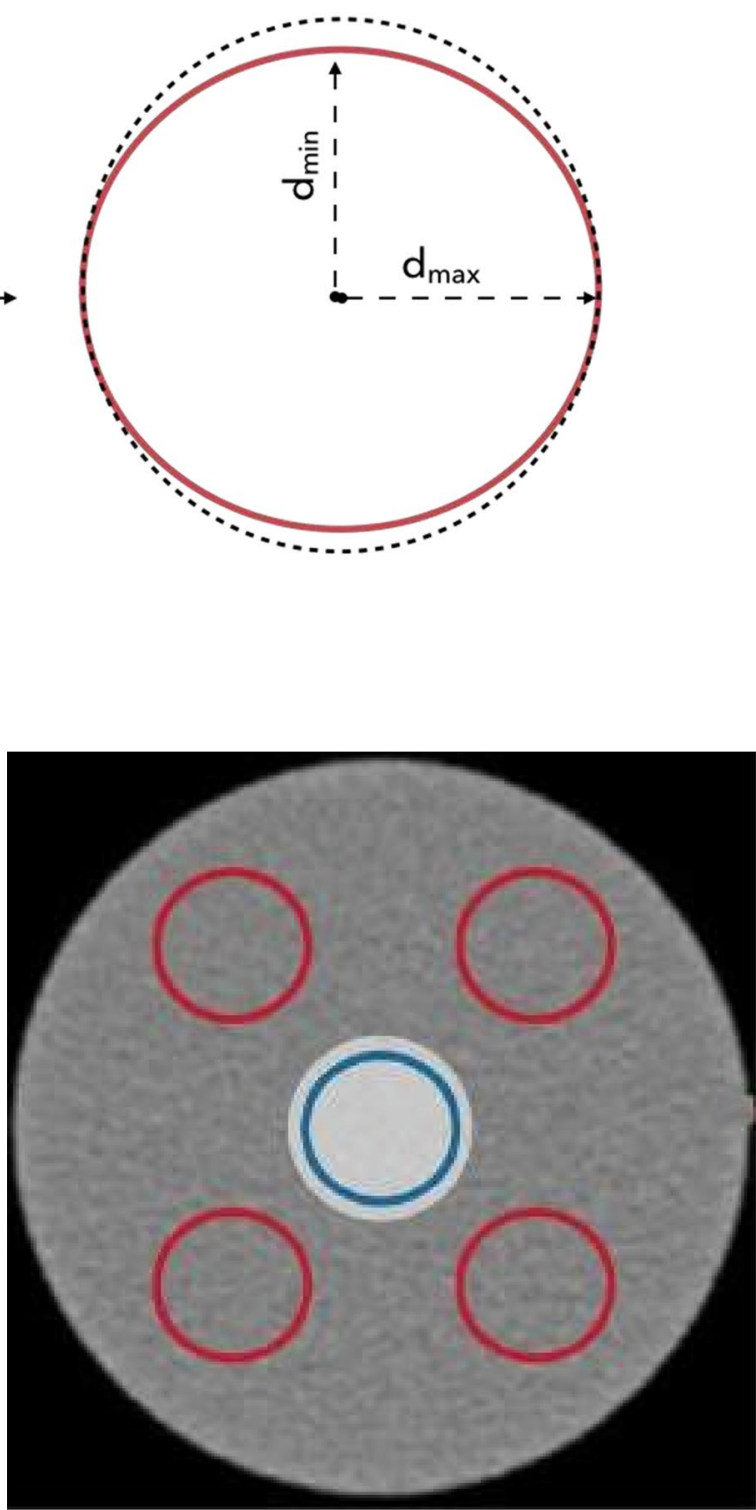

Fig. 4 Regions of interest of each hydroxyapatite concentration inserts

obtained by averaging five FWHM values along the vertical axis (Fig. 5).

\subsection{Statistical Analysis}

Each measurement was obtained by repeating this procedure in 10 continuous reconstructed slices in the central region. A t-test was used to assess differences between the two CBCT systems. Results were considered statistically significant at $p<0.05$. Statistical analysis was performed using R Statistical software, version 3.3.3. 

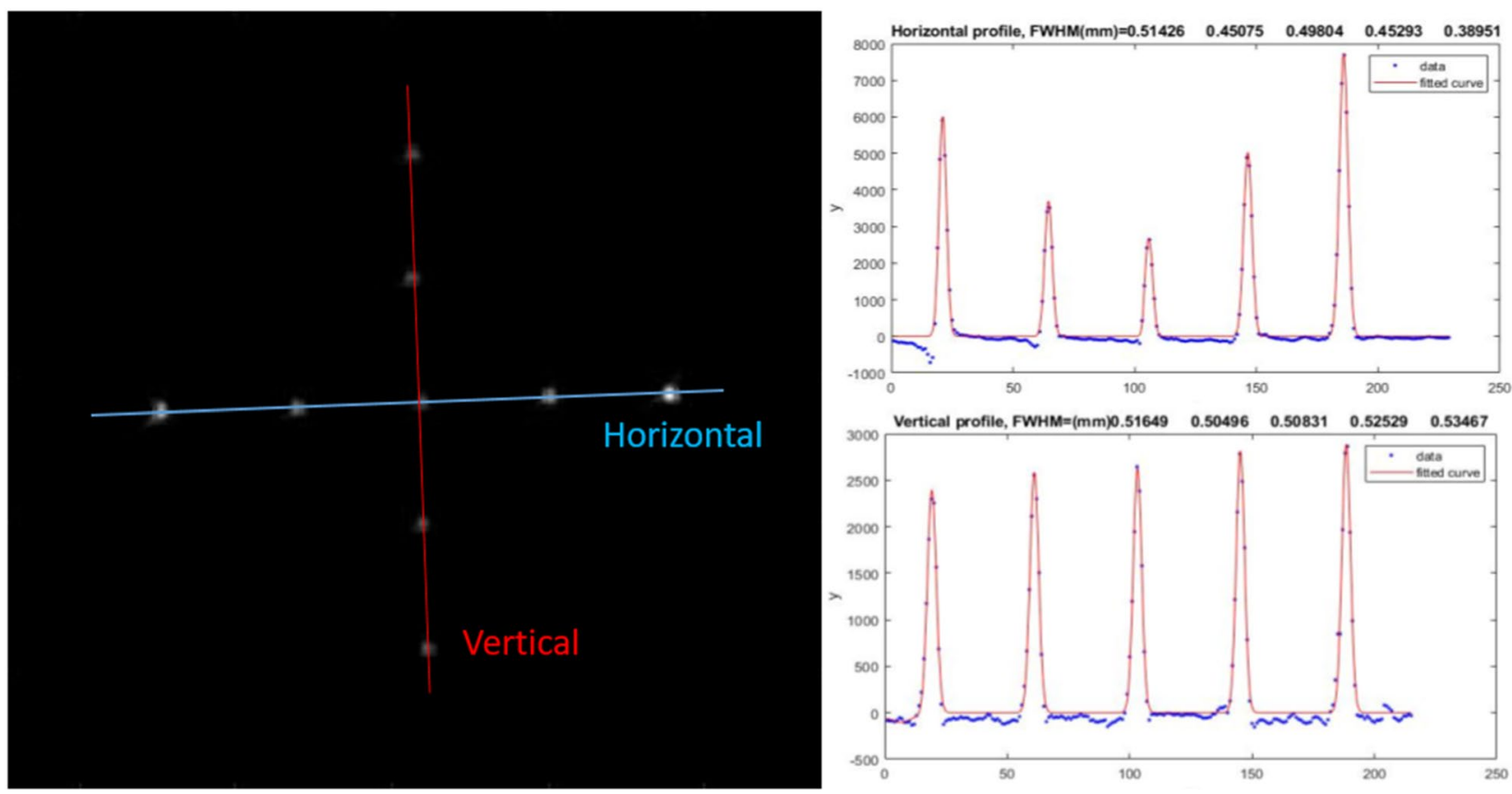

Fig. 5 The spatial resolution evaluation of the proprietary wire phantom: The horizontal direction is to evaluate FWHM from different wire thicknesses (from left to right: 160, 120, 100, 140, $180 \mu \mathrm{m}$ ) and

the vertical direction is to calculate the mean value of spatial resolution (same $100 \mu \mathrm{m}$ wires)

Table 3 Comparisons of image quality between two systems and factors related [13, 14]

\begin{tabular}{|c|c|c|c|}
\hline & System A & $\begin{array}{l}\text { Prototype } \\
\text { YMU-DENT-P001 }\end{array}$ & Can be affected by \\
\hline $\mathrm{CNR}^{*}$ & $100.29 \pm 2.31$ & $329.39 \pm 5.55$ & \multirow{2}{*}{$\begin{array}{l}\text { Dynamic range of the detector, exposure factors, bit depth, display setting, } \\
\text { graphic card's quality, noise, artifacts }\end{array}$} \\
\hline Linearity (R) & 0.99992 & 0.99928 & \\
\hline SNR* & $35.42 \pm 0.61$ & $159.85 \pm 3.88$ & \multirow[b]{2}{*}{$\begin{array}{l}\text { Three sources of noise: } \\
\text {-Quantum noise; } \\
\text { •Electronic noise; } \\
\bullet \text { Noise introduced by the reconstruction process } \\
\text { Be affected by intensity of X-ray source, circuit, detector design, number of } \\
\text { projections, exposure time, reconstruction algorithm, etc }\end{array}$} \\
\hline Uniformity $(\mathrm{U})^{*}$ & $30.59 \pm 2.32$ & $36.34 \pm 0.35$ & \\
\hline Spatial resolution* & $0.5179 \mathrm{~mm}$ & $0.2446 \mathrm{~mm}$ & $\begin{array}{l}\text { Focal spot size, detector element size, number of projections, smoothing filter, } \\
\text { reconstructed voxel size, reconstruction algorithm }\end{array}$ \\
\hline Diameter difference* & $1.59 \pm 0.04 \mathrm{~mm}$ & $1.14 \pm 0.005 \mathrm{~mm}$ & \multirow{2}{*}{$\begin{array}{l}\text { Distortion correction, center of rotation correction, flat field correction, position } \\
\text { of the reconstructed slice, radiation beam geometry, reconstruction algorithm, } \\
\text { the overlap of treatment and imaging center }\end{array}$} \\
\hline Distortion* & $0.285 \pm 0.003 \mathrm{~mm}$ & $0.03 \pm 0.002 \mathrm{~mm}$ & \\
\hline
\end{tabular}

$* \mathrm{p}<0.05$. CNR: Contrast-to-noise ratio; SNR: Signal-to-noise ratio

\section{Results}

\subsection{SNR, U, Distortion, and CNR}

Table 3 lists our analysis results. The CNR obtained from the prototype system exceeded that of system A by more than three times. The SNR and U obtained from the prototype system were significantly higher than those of the commercial system A $(p<0.05)$. These results demonstrate that the
YMU-DENT-P001 prototype outperformed the commercial system in terms of noise.

The actual diameter of the phantom was $32 \mathrm{~mm}$; however, the sizes estimated using reconstructed images from the edge of the phantom varied as follows: system A $(33.5903 \pm 0.004 \mathrm{~mm})$ and prototype system $(33.1437 \pm 0.005 \mathrm{~mm})$. The roundness distortion of the prototype $(0.03 \mathrm{~mm})$ was significantly lower than that of system A $(0.285 \mathrm{~mm})(\mathrm{p}<0.05)$. 


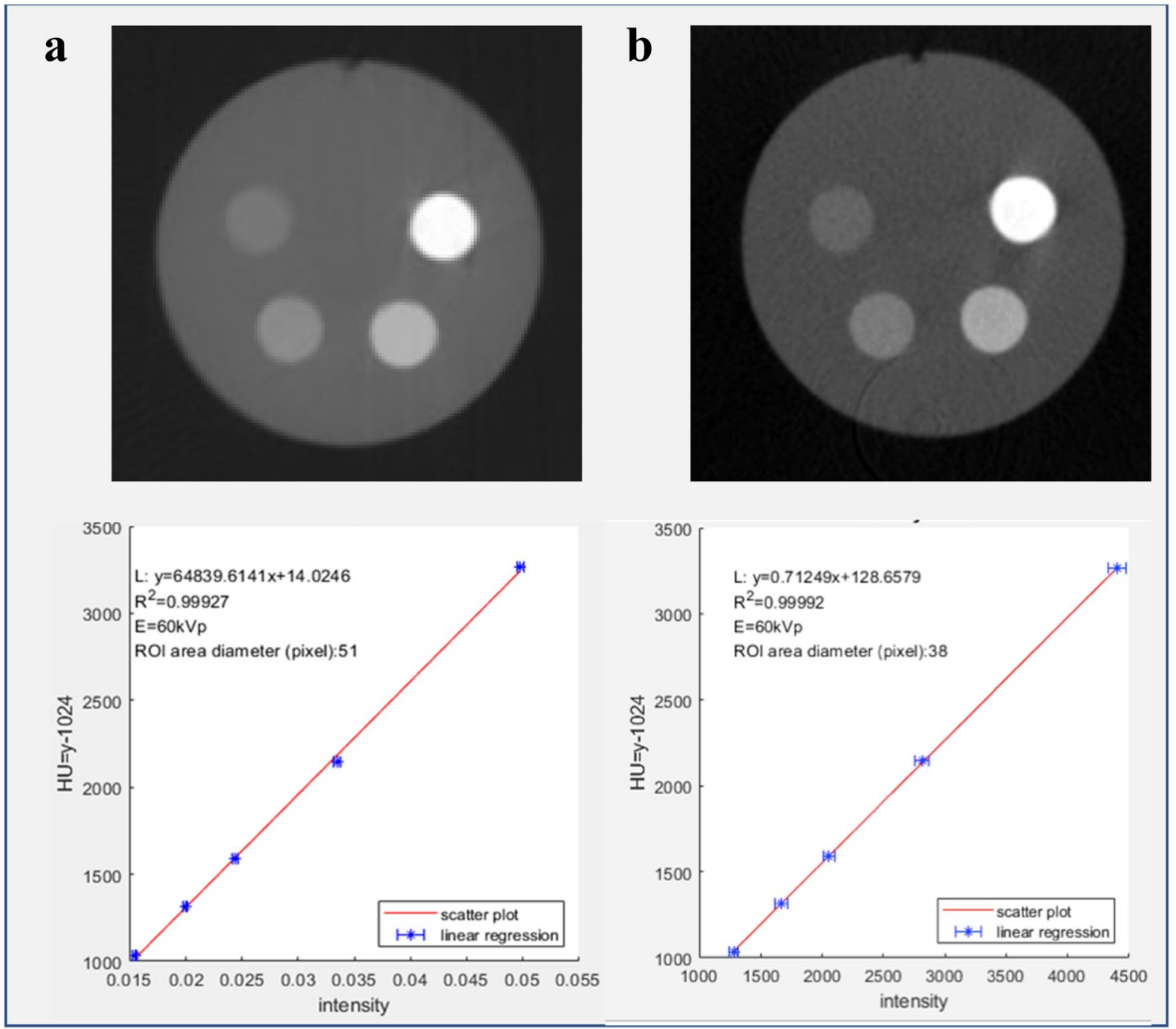

Fig. 6 Central slice of reconstructed HA phantom and analysis's results. a: HA phantom of prototype YMU-DENT-P001 system. b: HA phantom of system A's results

\subsection{Linearity in the Relationship Between Image Intensity and Density}

The correlation coefficients between image intensity and bone mineral density (BMD) in both systems was close to 1.0 (Fig. 6). The linear transfer function described in Eq. (5) can be used to translate grey-scale levels to HU for both systems.

\subsection{Spatial Resolution}

Figure $7 \mathrm{a}$ and $\mathrm{b}$ respectively present reconstructed images of the proprietary wire phantom used in the prototype and system A. We can clearly see nine points along the two-axes in the shape of a cross. The profile in the horizontal direction presents five PSFs of wires measuring 160, 120, 100, 140, and $180 \mu \mathrm{m}$. The profile in the vertical direction presents PSFs of five wires of the same size $(100 \mu \mathrm{m})$ by which to derive the mean spatial resolutions. The mean spatial resolution (FWHM) of the prototype was superior to that of system A $(\mathrm{p}<0.05)$, as shown in Table 4.

\section{Discussion}

In this study, we selected several high-precision test phantoms of tissue or water equivalent materials with known characteristics. This made it easy to perform constancy and acceptance tests on the CT imaging systems. QRM MicroCT Water, QRM Micro-contrast scale and Micro-CT HA are manufactured specifically for standardizing two or more CT imaging systems. We also created a proprietary wire phantom using a 3D-printer equipped with small acupuncture needles. We selected the smallest needle $(100 \mu \mathrm{m})$ as this is smaller than the smallest reported voxel size of system $\mathrm{A}(125 \mu \mathrm{m})$ and at the voxel size limit of the prototype 

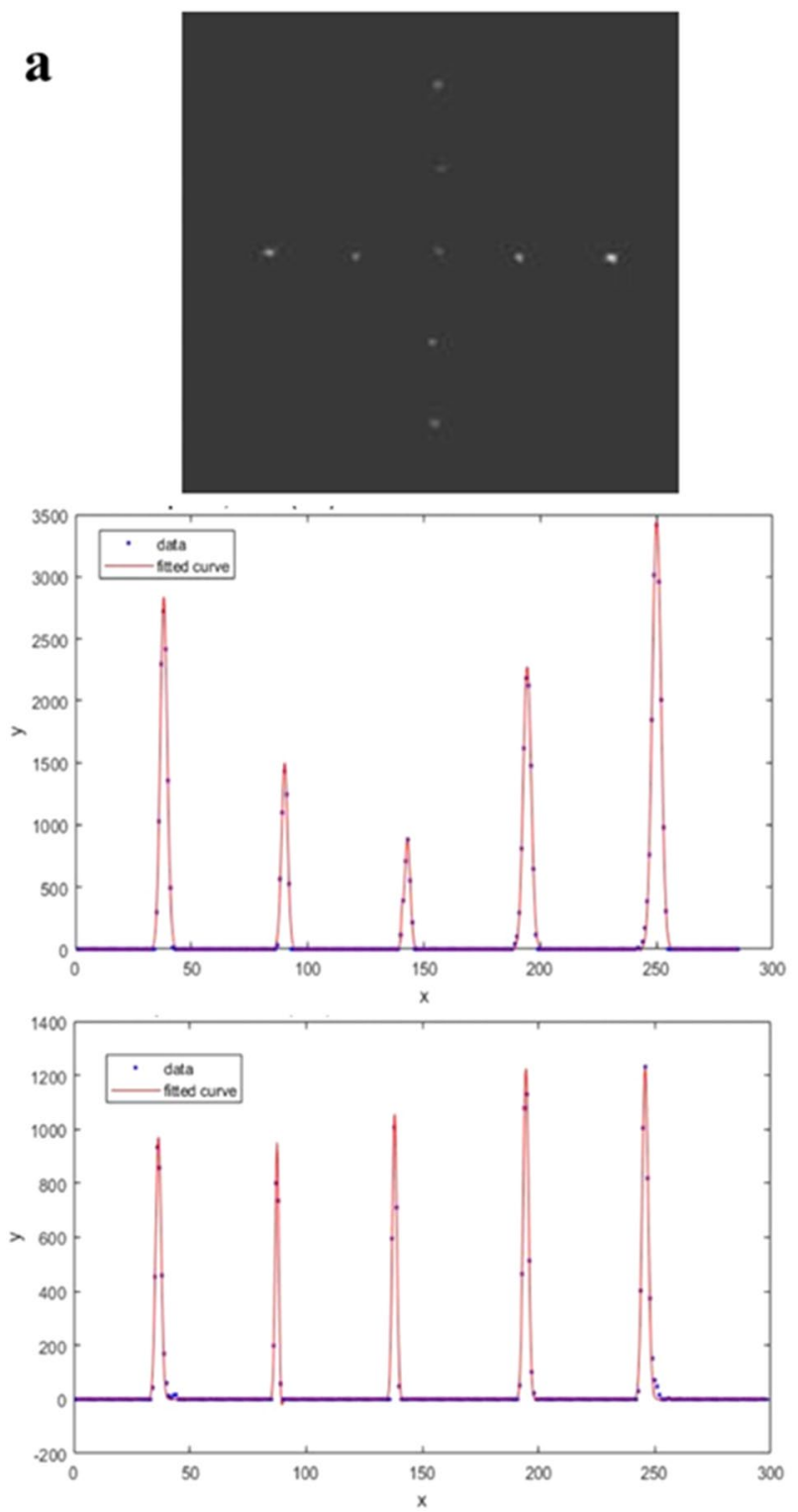

b
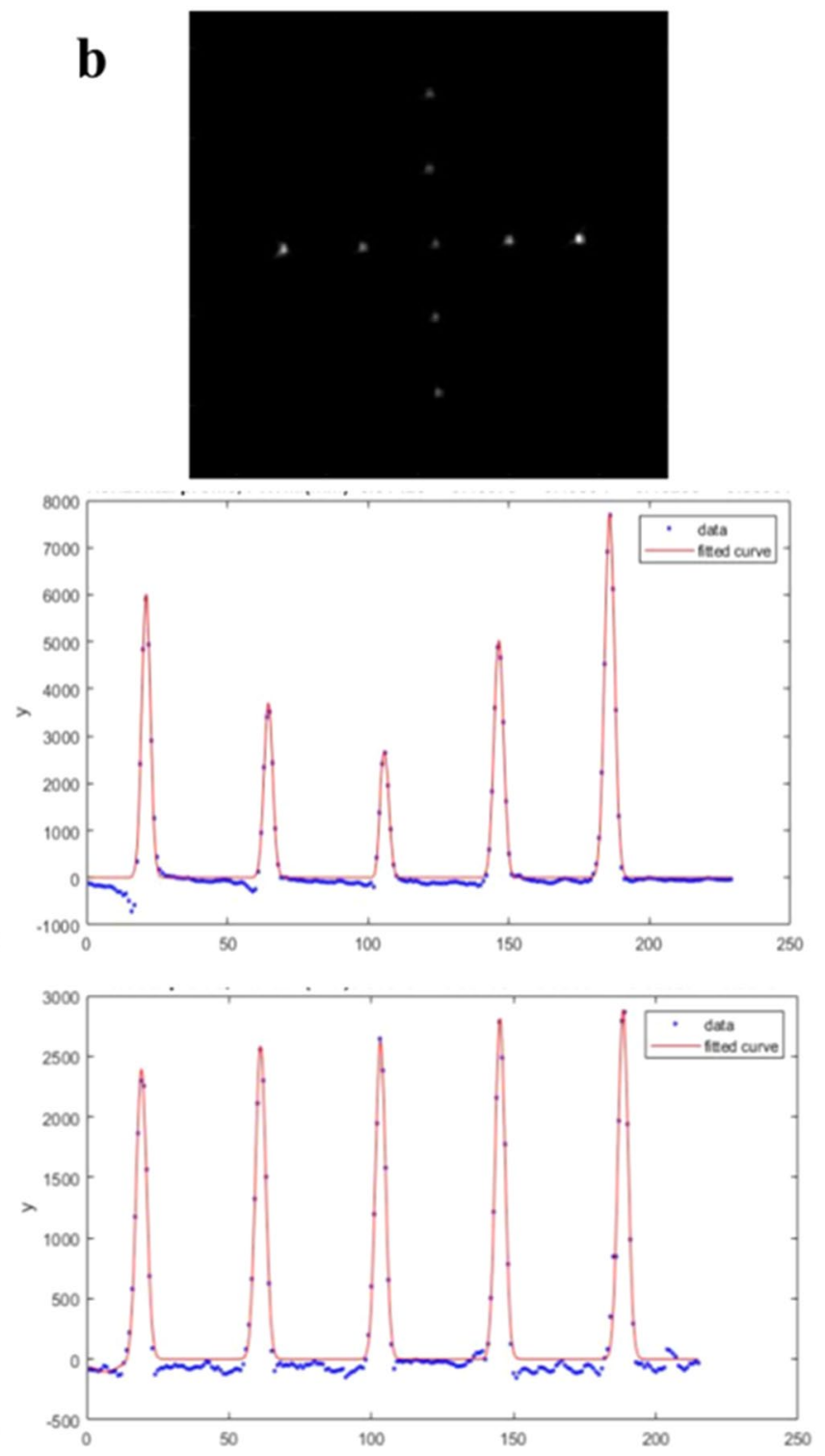

Fig. 7 Central slice of the reconstructed image of the proprietary wire phantom and analysis's results. a: Results of prototype YMU-DENT-P001 system. b: system A's results

$(100 \mu \mathrm{m})$. The phantom was small enough to ensure that it remained entirely within the field of view, thereby eliminating artifacts related to truncation and errors in voxel density values. The objective assessment of image quality using reproducible parameters such as $\mathrm{SNR}, \mathrm{CNR}$, U, distortion, and spatial resolution helps simplify the work of technicians involved in quality control of CBCT and researchers in comparing different systems [15].

The optimization of CT systems in accordance with the intended applications is inevitably a trade-off between resolution, noise, exposure settings, the speed of the acquisition stage, and cost. The noise that degrades radiographic images can be traced to a variety of sources: (1) quantum noise corresponding to the inherent randomness in the emission and detection of photons; (2) electronic noise related to circuitry in the image acquisition stage; and (3) noise introduced during the reconstruction process [14]. The relative amount of noise can be reduced by increasing X-ray exposure and maximizing the SNR when adjusting the source energy [13]. In the current study, we sought to eliminate the effects of source energy and window display settings in our comparison of image quality. The size of the detector elements in the two systems was not far different: system A $(0.15 \mathrm{~mm})$ and prototype $(0.139 \mathrm{~mm})$. This difference corresponds to $7.3 \%$ better for the prototype system. Nonetheless, the SNR of the prototype exceeded that of system 
Table 4 The average spatial resolution of the prototype DENT-P001 and system A through 10 slices

\begin{tabular}{llll}
\hline System & Phantom & $\begin{array}{l}\text { YMU-DENT-P001 } \\
\text { without binning }\end{array}$ & System A \\
Item & Wire thickness & FWHM $(\mu \mathrm{m})$ & FWHM $(\mu \mathrm{m})$ \\
\hline Horizontal & $100 \mu \mathrm{m}$ & 281.02 & 192.96 \\
profile & $120 \mu \mathrm{m}$ & 305.97 & 210.81 \\
& $140 \mu \mathrm{m}$ & 345.27 & 248.59 \\
& $160 \mu \mathrm{m}$ & 375.04 & 274.73 \\
& $180 \mu \mathrm{m}$ & 417.11 & 295.98 \\
Vertical & $100 \mu \mathrm{m}$ & 272.80 & 516.49 \\
profile & $100 \mu \mathrm{m}$ & 239.11 & 504.96 \\
& $100 \mu \mathrm{m}$ & 264.20 & 508.31 \\
& $100 \mu \mathrm{m}$ & 240.27 & 525.29 \\
& $100 \mu \mathrm{m}$ & 206.63 & 534.87 \\
Vertical mean* & & 244.60 & 517.98 \\
\hline
\end{tabular}

$* \mathrm{p}<0.05$

A by five times. This means that individual detectors in the prototype system detected the optimal number of photons to reduce noise, which demonstrates the efficiency of hardware set-up (X-ray tube or detectors) and operation system of the prototype.

Our results from the prototype YMU-DEN-P001 revealed the benefits of hardware improvements as well as the effectiveness of image processing, including denoising and artifact reduction. Our prototype system uses a number of correction methods developed for X-ray spectra, HU calibration, and artifact reduction (Table 2). However, denoise can result in blurring and make the reconstructed circle become enlarged. For that reason, the diameter of reconstructed image from the edge of the phantom is higher than the actual diameter of the phantom. The prototype system presented the size and geometry of captured structures with a higher degree of accuracy, due perhaps to a smaller voxel size. The effectiveness of the prototype reconstruction and image processing software was further demonstrated by the high degree of contrast, which can be so easily compromised by noise reduction or filtering algorithms.

The SNR values suggest that the distribution of pixel values in the prototype was more convergent in the prototype system than in system A. Nonetheless, the presence of many pixel value outliers affected the $U$ value. Note that despite lower SNR values, system A demonstrated superior performance in terms of U. Nevertheless, the standard deviation of $U$ values among axial slices revealed considerable uniformity (0.35) across the axial planes of the prototype, far exceeding that of system A (2.32).

CNR is a quantitative measurement of low-contrast resolution, indicating the system's ability to differentiate a signal from the background, such that a higher CNR value indicates superior performance in differentiating among tissue types. The correlation coefficient between HA concentration and pixel value did not vary between the systems $(\mathrm{R} \approx 1)$; however, the prototype system outperformed system $\mathrm{A}$ in terms of CNR, probably due to superior control over noise. This means the recognition performance of the prototype was better when the background and structures presented a similar attenuation coefficient.

The size of the ROIs used for the analysis of SNR, U, and CNR was carefully selected. Our aim was to make it large enough to eliminate the effects of local inhomogeneity and small enough to limit the effect of artifacts. In testing the linearity of the attenuation coefficient, we limited the size of the ROIs chosen in each cylinder to avoid the blurring of edges. The prototype system also outperformed system A in resisting deformation. The processes of distortion correction or center of rotation correction might be applied differently. However, such methods are beyond the scope of current study. Other factors related to X-ray tube quality, artifacts (X-ray scatter, ring artifact, beam-hardening, truncation, mental artifact) should also be considered in future studies.

Spatial resolution is a system-level performance metric affected by the size of the detector element, the size of the focal spot, and the source to detector distance as well as the methods, filters, and voxel size used in reconstruction [6]. The two systems did not differ considerably in terms of detector element size or focal spot size. The superior spatial resolution of the prototype $(0.2446 \mathrm{~mm})$ compared with system A $(0.5179 \mathrm{~mm})$ can perhaps be explained by the SOD/ OID ratio: prototype (2.26) vs. system A (2.05). It might also be attributed to the fact that the source-to-detector distance of the prototype $(620 \mathrm{~mm})$ exceeded that of system A $(520 \mathrm{~mm})$, thereby permitting higher geometric magnification. The use of modulation transfer function (MTF) to quantitatively assess the spatial resolution of CBCT systems could be used to verify that the values obtained in real-world clinical environments are consistent with the values reported by the manufacturer.

\section{Conclusions}

Within the scope of the current study, the proposed system and system A both presented acceptable image quality; however, the prototype outperformed system A in terms of SNR, CNR, spatial resolution, and distortion. The proprietary phantom fabricated in this study suggests a simplified approach to phantom design for the periodic assessment and quality control of CBCT systems.

Acknowledgements This research instrument design and development was supported by funding from the Ministry of Science and Technology, Taiwan, R.O.C grants: MOST 107-2328-8-010-003. 
Author Contribution All authors read and approved the final manuscript. All authors contributed to the study conception and design: Trang Thi Ngoc Tran: Design the experiment, design customized phantom, collect data, analysis, writing manuscript. David Shih-Chun Jin: Design the experiment, collect data, software for image analysis, data interpretation. Kun-Long Shih: Collect data from experiment, analyze system A's parameters and images quality. Jyh-Cheng Chen: Supervisor, define the concept of the work, design the experiment, review manuscript. Ming-Lun Hsu: Supervisor, design the experiment, review manuscript.

Funding This research instrument building was supported by funding from the Ministry of Science and Technology, Taiwan, R.O.C grants: MOST 107-2328-8-010-003.

Data Availability Data available on request from the authors.

Code Availability Not applicable.

\section{Declarations}

Conflict of interest The authors have no conflict of interest to disclose.

Open Access This article is licensed under a Creative Commons Attribution 4.0 International License, which permits use, sharing, adaptation, distribution and reproduction in any medium or format, as long as you give appropriate credit to the original author(s) and the source, provide a link to the Creative Commons licence, and indicate if changes were made. The images or other third party material in this article are included in the article's Creative Commons licence, unless indicated otherwise in a credit line to the material. If material is not included in the article's Creative Commons licence and your intended use is not permitted by statutory regulation or exceeds the permitted use, you will need to obtain permission directly from the copyright holder. To view a copy of this licence, visit http://creativecommons.org/licenses/by/4.0/.

\section{References}

1. Mozzo, P., Procacci, C., Tacconi, A., Martini, P. T., \& Andreis, I. A. (1998). A new volumetric CT machine for dental imaging based on the cone-beam technique: preliminary results. European Radiology, 8(9), 1558-1564.

2. Popescu, D., \& Laptoiu, D. (2016). Rapid prototyping for patientspecific surgical orthopaedics guides: a systematic literature review. Proceedings of the Institution of Mechanical Engineers. Part H, 230(6), 495-515.
3. Francis, Z., Louise, R., \& Mark, F. M. (2010). Image quality assessment tools for optimization of CT images. Radiography, 16(2), 147-153.

4. Liang, X., Jacobs, R., Hassan, B., Li, L., Pauwels, R., Corpas, L., et al. (2010). A comparative evaluation of cone beam computed tomography (CBCT) and multi-slice CT (MSCT) Part I. On subjective image quality. European Journal of Radiology, 75(2), 265-269.

5. Dillenseger, J. P., Matern, J. F., Gros, C. I., Bornert, F., Goetz, C., Le Minor, J. M., et al. (2015). MSCT versus CBCT: evaluation of high-resolution acquisition modes for dento-maxillary and skullbase imaging. European Radiology, 25(2), 505-515.

6. Pauwels, R., Araki, K., Siewerdsen, J. H., \& Thongvigitmanee, S. S. (2015). Technical aspects of dental CBCT: state of the art. Dentomazillofac Radiol, 44, 20140224.

7. Goldman, L. W. (2007). Principles of CT: radiation dose and image quality. Journal of Nuclear Medicine Technology, 35(4), 213-225.

8. Pauwels, R., Beinsberger, J., Stamatakis, H., Tsiklakis, K., Walker, A., Bosmans, H., et al. (2012). Comparison of spatial and contrast resolution for cone-beam computed tomography scanners. Oral Surgery, Oral Medicine, Oral Pathology, and Oral Radiology, 114, 127-135.

9. Kim, D. G. (2014). Can dental cone beam computed tomography assess bone mineral density? J Bone Metab, 21(2), 117-126.

10. Magill, D., Beckmann, N., Felice, M. A., Yoo, T., Luo, M., \& Mupparapu, M. (2018). Investigation of dental cone-beam CT pixel data and a modified method for conversion to Hounsfield unit (HU). Dento Maxillo Facial Radiology, 47(2), 20170321.

11. Kalender, W. A., Deak, P., Kellermeier, M., van Straten, M., \& Vollmar, S. V. (2009). Application- and patient size-dependent optimization of x-ray spectra for CT. Medical Physics, 36, 993-1007.

12. QRM, micro-CT \& PET-/SPECT phantoms. Available from https://www.qrm.de/en/overview-pages/micro-ct-pet-spect-phant oms/ [Accessed 22 December 2020].

13. International Organization for Standardization (2002). Non destructive testing - Radiation methods for computued tomography - ISO/DIS standard No.15708, part 1-2-3. Retrived from https://www.iso.org/standard/72254.html

14. American National Standard (2003). Measurement of Out-ofroundness - ANSI B89.3.1-1972. Retrived from https://webst ore.ansi.org/standards/asme/ansiasmeb891972r2003.

15. Las Heras Gala, H., Torresin, A., Dasu, A., Rampado, O., Delis, H., Hernández Girón, I., et al. (2017). Quality control in conebeam computed tomography (CBCT) EFOMP-ESTRO- IAEA protocol (summary report). Physica Medica, 39, 67-72. 